\title{
History taking
}

\author{
Mr. Jerrish A Jose ${ }^{\mathrm{a}}$ \\ Consultant in Physiotherapy and Ergonomics, RECOUP Neuromusculoskeletal Rehabilitation Centre, Bangalore, \\ INDIA
}

\begin{abstract}
History taking is the staple essential backbone for making an accurate diagnosis; it will enable the clinician to make a provisional diagnosis in most of the cases. The following rules should be taken in to consideration for while taking history. Objectives. The history taking will enable the clinician to organise the patient's story, filter the information which links to common musculoskeletal disorders by means of clinical reasoning, to fully understand the patient's present health status and to form a provisional diagnosis.
\end{abstract}

Keywords: Pain, Medical History, Occupational History, Family History

\section{Allow enough time}

The patient should feel the clinician has time availability and appropriate body language to allow the story to spread out; otherwise there are chances of missing out crucial symptoms. In addition to the details of the injury, try to look for the possible causes of injury. A minimum of 30 minutes is usually required to assess the new patient with the typical presentation, however in case of composite chronic cases clinician may need to avail 1 hour.

\section{Know the work}

Knowing the technical demands of a patient work during consultation is the added advantage for the clinician, as this engenders patient confidence. Moreover knowing biomechanics and techniques of a peculiar work tasks can assist clinician in making provisional diagnosis and unveiling predisposing factors.

\section{Obtain an accurate description of Symptoms}

Common musculoskeletal symptoms include pain, swelling, instability and loss of function and their precise description is essential.

\subsection{Pain}

\subsubsection{Location}

Mark the exact location of the pain, a comprehensive knowledge of the surface anatomy will enable you to differentiate the structures likely to be involved. If the pain is vague or varies from site to site, think the possibility of referred pain.

\subsubsection{Onset}

Speed of the onset helps in understanding whether pain is of acute or overuse in nature.

\subsubsection{Severity}

It may be classified in to mild, moderate or severe. Measure the severity of the pain instantly after the injury and later on.

Corresponding author, Email ID: jerrish@recoup.in

${ }^{1}$ Address: \#312, 80 Feet Road, 10th Block, Further Extension of Anjanapura Layout, Bangalore-560062 


\subsubsection{Irritability}

It indicates the amount of work required to evoke pain and amount of time it takes to settle subsequently. It influences the nature and aggressiveness of the examination and the treatment.

\subsubsection{Aggravating/ Relieving Factors}

It indicates whether certain activities or positions aggravate/ relieve the pain.

\subsection{Swelling}

Prompt swelling after an injury may suggest a severe injury such as a fracture or ligamentous injury. Measure the degree of the swelling as mild, moderate or severe and subsequent changes in the swelling.

\subsection{Instability}

A history of giving way or feeling of instability usually indicates a ligamentous injury.

\subsection{Function}

It is helpful to know whether the patient was able to continue work without any problems following the injury, was able to work with limitation or was not able to work at all. Subsequent changes in function with time should also be noted as it is helpful to presume the recovery or prognosis.

\section{History of a previous similar injury}

As previous injury is a major risk factor for recurrence, details of previous similar injury, detail of all treatment received and maintenance treatment or exercises executed following initial rehabilitation must be described.

\subsection{Family History}

Family history gives the understanding of any prevalence of genetic musculoskeletal disorders such as arthritis and others. Gathering a complete and accurate family medical history is becoming more important as genetic medicine explains more diseases.

\subsection{Occupational History}

Occupational history gives the understanding of probable risk factor for the development of overuse musculoskeletal disorders. Inappropriate equipment handling due to poor execution technique or equipment itself may predispose to either acute or chronic musculoskeletal disorders. Psychological factors such as peer pressure, reduced motivation etc. may exacerbate or influence the current status of injury. Symptoms such as excessive fatigue, recurrent episodes of pain or illness, persistent soreness and stiffness suggests act of over working.

\subsection{Medical History}

It gives you the understanding of the severity, intensity and acuteness of the disorders. The physician need to have complete details about starting date or time, how the condition started, how the symptoms changed over the period of time, about the acuteness of the problem, etc. As musculoskeletal symptoms are not always work or activity related physician should know the general health and fitness level to eliminate the diseases which masquerade as musculoskeletal disorders. All these information helps to plan the treatment, exercise prescription and guide the patients about the treatment duration and other information.

\section{References}

[1] P. Brukner and K. Khan, Principles of Diagnosis: Clinical Assessment, in: Clinical Sports Medicine, $3^{\text {rd }}$ ed., McGraw-Hill, Sydney, London, 2006, pp. 108-119. 